\title{
Mejora del aprendizaje discriminativo en niños: consecuencias diferenciales y administración manual de diferentes formas de refuerzo
}

\author{
Angeles F. Estévez ${ }^{1}$, Isabel Carmona ${ }^{1}$, Laura Esteban ${ }^{1}$ y Victoria Plaza ${ }^{2 *}$ \\ ${ }^{1}$ Universidad de Almería (España). \\ ${ }^{2}$ Universidad Autónoma de Chile (Chile).
}

\begin{abstract}
Resumen: Diversos estudios han mostrado que el aprendizaje discriminativo puede ser mejorado mediante la asignación de una consecuencia única para cada asociación que ha de ser aprendida (procedimiento de consecuencias diferenciales, PCD). El objetivo de este estudio fue comprobar s el PCD mejoraría el aprendizaje de discriminaciones simbólicas en niños de 5 y 7 años de edad, mediante una tarea de lápiz y papel en la que se usaron diferentes tipos de entrenamiento: 1) administración de reforzadores tras las respuestas correctas $(+), 2)$ retirada de reforzadores tras los errores $(-)$, o 3) una combinación de ambas (+/-). En los Experimentos 1a y 1b se utilizaron reforzadores primarios y secundarios, mientras que en los Experimentos $2 \mathrm{a}$ y $2 \mathrm{~b}$ se utilizaron solo reforzadores primarios. Los participantes mostraron una mejor ejecución en las tareas cuando se administraron consecuencias diferenciales independientemente del tipo de reforzador (primario y secundario o sólo primario) y del tipo de entrenamiento empleado $(+$ -, o +/-). Estos resultados se suman a los encontrados en estudios previos (Martínez, Estévez, Fuentes y Overmier, 2009; Martínez, Flores, GonzálezSalinas, Fuentes y Estévez, 2013) demostrando, además, que el efecto beneficioso del PCD sobre el aprendizaje discriminativo es independiente de medio por el cual presentamos los estímulos y las consecuencias, lo que facilita su posible utilización en contextos aplicados.

Palabras clave: Procedimiento de consecuencias diferenciales; aprendizaje discriminativo; tipos de reforzamiento; niños.
\end{abstract}

\section{Introducción}

El aprendizaje discriminativo es un tipo de aprendizaje muy común e importante en nuestra vida cotidiana, ya que nos permite discriminar entre diferentes situaciones y actuar de una forma adecuada. Por ejemplo, imaginemos que queremos enseñar a un niño a identificar el nombre de las notas musicales tras escuchar los diferentes tonos. Este aprendizaje discriminativo implica diferenciar entre los distintos sonidos y asociarlos con cada nombre en particular. Experimentos realizados en laboratorio han mostrado que cuando se administran consecuencias específicas para cada asociación estímulo-respuesta a aprender, dicho aprendizaje es más eficiente, es decir, se aprende antes y se cometen menos errores que cuando éstas se presentan de forma aleatoria (para una revisión, ver Estévez, 2005; López-Crespo y Estévez, 2013).

Los primeros datos que se obtuvieron al respecto fueron proporcionados por Trapold en 1970. En su estudio, expuso a un grupo de ratas a una tarea de discriminación condicional. Concretamente, realizó un experimento donde debían aprender a presionar una determinada palanca ante un estímulo concreto. Por ejemplo, ante un estímulo determinado (tono) debía ser presionada la palanca de la derecha (R1) y ante otro estímulo (clic) la palanca de la izquierda (R2). Tra-

* Dirección para correspondencia [Correspondence address]:

Victoria Plaza. Universidad Autónoma de Chile. 5 Poniente 1670 3460000 Talca (Chile). E-mail: mplazar@uautonoma.cl
Title: Improvement of discriminative learning in children: differential outcomes and manual administration of different types of reinforcement. Abstract: In recent years, several studies have demonstrated that discriminative learning is facilitated when each association to be learned is always followed by a unique outcome. This way of providing outcomes was called the differential outcomes procedure (DOP). The aim of the study reported here was to assess whether the DOP could improve learning of symbolic conditional discriminations in 5- and 7-year-old children when performing a paper-and-pencil task using different types of training in which: 1) reinforcers were given after correct choices $(+), 2)$ reinforcers were withdrawn when errors were made (-), or 3$)$ a combination of both (+/-). In Experiments $1 \mathrm{a}$ and $1 \mathrm{~b}$ secondary reinforcers were used along with primary reinforcers, while in Experiments $2 \mathrm{a}$ and $2 \mathrm{~b}$ only primary reinforcers were used. Participants showed better performance when differential outcomes were arranged regardless of the type of reinforcers (secondary plus primary or only primary) and the different strategies of reinforcement $(+,-$, or $+/-)$. These results add to those found by Martínez et al. $(2009,2013)$ and demonstrate that the beneficial effect of the DOP on discriminative learning is also independent of the way in which the stimuli and the outcomes are presented, facilitating its use in applied contexts.

Keywords: Differential outcomes procedure; discriminative learning; types of reinforcement; children.

pold observó un incremento en la adquisición y en la exactitud final de la tarea cuando la respuesta correcta R1 fue seguida por pellets y la respuesta correcta R2 por sacarosa en comparación a cuando se administró un mismo tipo de reforzador como, por ejemplo, sacarosa, tras ambas respuestas correctas. Este autor concluyó que se podría mejorar la ejecución en tareas de discriminación condicional cuando cada asociación a aprender fuera seguida de un único refuerzo específico. A esta forma de administración de los refuerzos, o consecuencias, se la denominó procedimiento de consecuencias diferenciales (PCD; Peterson y Trapold, 1980).

Investigaciones posteriores han mostrado los beneficios del PCD sobre el aprendizaje discriminativo, extendiendo los resultados de los estudios de Trapold a una amplia variedad de tareas, consecuencias y especies (para una revisión, ver López-Crespo y Estévez, 2013; Mok, Estévez y Overmier, 2010; Urcuioli, 2005). Los estudios realizados en humanos sobre aprendizaje discriminativo han mostrado que el PCD también produce un incremento en la velocidad de adquisición y/o una mejora en la ejecución de la tarea, en diferentes poblaciones como niños y adultos sin discapacidad (p.e., Easton, 2004; Estévez y Fuentes, 2003; Estévez, Fuentes, Mari-Beffa, González y Álvarez, 2001; Estévez et al., 2007; Legge y Spetch, 2009; Maki, Overmier, Delos y Gutman, 1995; Miller, Waugh y Chambers, 2002; Mok y Overmier, 2007; Mok, Thomas, Lungu y Overmier, 2009), adultos con Síndrome de Prader-Willi (Joseph, Overmier y Thompson, 1997), niños nacidos con gran prematuridad (Martínez 
et al., 2012) y niños y adultos con Síndrome de Down (Estévez, Overmier, Fuentes y González, 2003).

En todos estos estudios, las consecuencias administradas tras las respuestas correctas han sido reforzadores positivos; sin embargo, en nuestra vida cotidiana, las consecuencias que recibimos también implican la retirada de los refuerzos (por ejemplo, una infracción de tráfico suele ir acompañada por una pérdida de puntos de nuestro carnet de conducir). En este sentido, dos investigaciones recientes se diseñaron con el objetivo de comprobar si ambos tipos de entrenamiento (la ganancia y/o la pérdida de objetos valorados) podrían afectar a la ejecución de tareas de discriminación bajo consecuencias diferenciales en niños de 5 y 7 años de edad, respectivamente (Martínez, Estévez, Fuentes y Overmier, 2009; Martínez, Flores, González-Salinas, Fuentes y Estévez, 2013). Para ello utilizaron una tarea de igualación demorada a la muestra en la que los participantes debían aprender asociaciones de figuras abstractas utilizando, además, dos tipos diferentes de consecuencias (reforzadores primarios y secundarios $v$ s. sólo reforzadores primarios). En la condición diferencial, los tres tipos de entrenamiento que se evaluaron consistieron en 1) la administración de un reforzador específico tras cada respuesta correcta (grupo "+"), 2) la retirada de un reforzador específico tras cada respuesta incorrecta (grupo “-”), o 3) la utilización de ambos procedimientos simultáneamente (grupo “+/-”). Los tres grupos de la condición no diferencial fueron similares con la excepción de que los refuerzos que se ganaban y/o perdían se administraron de manera aleatoria. Los resultados mostraron que, en general, los niños de 5 y 7 años que fueron entrenados con el PCD, aprendieron y recordaron mejor las discriminaciones simbólicas complejas bajo los tres tipos de entrenamiento que aquellos a los que no se les reforzaron diferencialmente. De la misma manera, el efecto beneficioso que el PCD tiene sobre el aprendizaje discriminativo simbólico fue también independiente de la consecuencia utilizada. Concretamente, en ambos estudios se administraron bien reforzadores secundarios junto con primarios (fichas de colores + golosinas/juguetes) o solo reforzadores primarios (golosinas/juguetes), lo que demuestra que el efecto no se ve influenciado por el hecho de que las consecuencias inmediatas a las respuestas sean o no reforzadores primarios.

Con respecto a los tres tipos de entrenamiento, los resultados mostraron que los niños de 5 años presentaron una mejor ejecución en la tarea cuando se utilizó el tipo de entrenamiento “_", es decir, cuando perdieron reforzadores tras sus errores. Los autores atribuyen estos resultados al hecho de que los niños de este grupo de edad son más sensibles a la pérdida de reforzadores, posiblemente porque aún no han desarrollado estrategias cognitivas que les permitan enfrentarse al estado de frustración que les supone la retirada de algo valioso para ellos. Esta explicación estaría sustentada por los datos de investigaciones que muestran que a esta edad existe un menor desarrollo de la corteza frontal, estructura implicada en la generación de este tipo de estrategias cognitivas (p.e., Lewis, Lamm, Segalowith, Stieben y Ze- lazo, 2006; Posner y Rothbart, 2009). El estado de frustración generado por la pérdida de reforzadores produciría un incremento en el nivel de arousal que tendría como consecuencia la optimización de la ejecución del grupo de niños de cinco años tanto en la condición diferencial como en la no diferencial, lo que estaría en consonancia con estudios previos que muestran que un incremento moderado en el nivel de arousal produce un efecto beneficioso en la ejecución de distintos tipos de tareas (p.e., Walters y Parke, 1964). Por el contrario, un mayor desarrollo de la corteza frontal en los niños de 7 años, les permitiría desarrollar estrategias cognitivas más adecuadas para enfrentar las posibles consecuencias emocionales negativas derivadas de la retirada del reforzador, por lo que se reduciría la necesidad de activar otros procesos para tratar con dichas emociones negativas que podrían, a su vez, influir en la ejecución de la tarea.

Cabe señalar que sólo en los dos estudios realizados por Martínez y colaboradores $(2009,2013)$ se ha explorado si el efecto beneficioso que la utilización de consecuencias diferenciales se ve modulado por el tipo de entrenamiento así como por los reforzadores empleados (primarios $v$ s. primarios y secundarios). Además, en ambos estudios tanto la presentación de la secuencia de estímulos como de los reforzadores secundarios fue informatizada. En la presente investigación nos preguntamos si encontraríamos resultados similares a los obtenidos por Martínez y colaboradores (2009, 2013) utilizando una tarea de lápiz y papel y administrando los reforzadores de forma manual. El objetivo principal de este estudio fue, por tanto, comprobar si el efecto beneficioso que tiene la utilización de consecuencias diferenciales en el aprendizaje discriminativo, no sólo es independiente del tipo de consecuencias y del tipo de entrenamiento empleado sino, también, del medio por el cual presentamos los estímulos y las consecuencias, lo que facilitaría la posible utilización del procedimiento en contextos aplicados (p.e., clínica, escuela, hogar).

\section{Experimentos 1a y $1 b$}

El objetivo de este experimento fue continuar investigando la influencia de diferentes tipos de reforzamiento sobre el efecto que produce el PCD en el aprendizaje discriminativo de niños de 5 y de 7 años de edad. Para ello se utilizó una tarea de igualación simbólica demorada a la muestra, similar a las empleadas por Martínez et al. (2009, 2013), con la salvedad de que en el presente estudio la tarea se presentó en formato papel.

\section{Método \\ Participantes}

Todos los niños que participaron en el estudio eran alumnos del C.E.I.P Lope de Vega (Almería, España). En el experimento 1 a participaron 54 niños con edades comprendidas entre 4 años y 11 meses a 5 años y 11 meses (23 niños 
y 31 niñas) y en el experimento $1 \mathrm{~b}, 36$ niños de entre 6 años y 11 meses a 7 años y 11 meses años de edad (19 niños y 17 niñas). Todos los niños tenían visión normal o corregida a la normalidad.

\section{Estimulos y materiales}

La tarea se administró a cada niño de forma individual. Al comenzar el experimento cada participante se sentaba frente al experimentador en una sala tranquila del colegio. Los estímulos, similares a los utilizados por Martínez et al. (2009, 2013), fueron distintos dibujos (unas tijeras, una flor, un rombo, una cruz, la letra griega gamma, dos figuras geométricas -una con forma de círculo y otra con forma de espiral-, y cuatro combinaciones de flechas) presentados en un cuaderno situado en una mesa ubicada frente a ellos. La medida de los estímulos fue de $5 \times 5 \mathrm{~cm}$, aproximadamente, y estaban dibujados en color negro sobre fondo blanco.

A la izquierda del experimentador se colocaron dos recipientes, uno rojo y otro verde. Tras una respuesta correcta, los niños recibían (o se les quitaba) una ficha roja o una verde, que tenían que depositar en el recipiente del color correspondiente. Los niños podían canjear estas fichas por comida o por juguetes al finalizar el experimento. La comida consistió en galletas, chicles y gominolas, que estaban colocados en una caja roja. Los juguetes eran ceras de colores, pegatinas y globos, y estaban situados en una caja verde. Las cajas estaban ubicadas cerca de los niños y fuera del alcance de su visión. A lo largo de todo el experimento, el experimentador controló la presentación de los estímulos, el registro de datos y la administración de consecuencias. Un se- gundo observador también anotaba las respuestas de los niños y las consecuencias administradas. El observador estaba sentado fuera de la línea de visión de los niños, pero lo suficientemente cerca como para ver claramente sus respuestas.

\section{Procedimiento}

Los participantes fueron asignados a dos grupos según su edad, 5 y 7 años. Se establecieron seis condiciones experimentales, como combinación de (i) la administración de consecuencias, de forma diferencial (D), en las que a cada emparejamiento correcto -o incorrecto- estímulo discriminativo-respuesta le correspondía una consecuencia específica (ganar -o perder- una ficha roja o verde), o no diferencial (ND), en el que tras cada respuesta correcta - o incorrectala consecuencia administrada fue aleatoria (ganar -o perderuna ficha verde o roja); y (ii) el tipo de reforzamiento administrado, que podía ser de tres tipos: “+”, que consistía en dar un reforzador secundario (ficha roja o verde) tras una respuesta correcta; "-”, que consistía en quitar una de las fichas que al inicio del experimento se entregaron a cada niño en esta condición experimental (20 fichas rojas y 20 verdes) tras una respuesta incorrecta; y "+/-", en el que se daba a los niños un reforzador secundario tras cada respuesta correcta y se les quitaba uno tras cada respuesta incorrecta (ver Tabla 1). Las fichas rojas y verdes actuaron como reforzadores secundarios, y la comida y los juguetes como reforzadores primarios. Los niños de cada grupo de edad, fueron asignados aleatoriamente a una de las seis condiciones experimentales.

Tabla 1. Condiciones experimentales establecidas en el Experimento 1a.

\begin{tabular}{|c|c|c|c|c|c|}
\hline \multirow[b]{2}{*}{ Consecuencia } & \multicolumn{2}{|c|}{ Estimulos } & \multirow[b]{2}{*}{$\mathrm{D}(+)$} & \multirow[b]{2}{*}{$\mathrm{D}(-)$} & \multirow[b]{2}{*}{$D(+/-)$} \\
\hline & Muestra & Comparación & & & \\
\hline & & & $\begin{array}{c}\text { Gana una ficha } \\
\text { roja }\end{array}$ & Nada & $\begin{array}{c}\text { Gana una ficha } \\
\text { roja }\end{array}$ \\
\hline & & & Nada & $\begin{array}{c}\text { Pierde una ficha } \\
\text { roja }\end{array}$ & $\begin{array}{c}\text { Pierde una ficha } \\
\text { roja }\end{array}$ \\
\hline & & & Nada & $\begin{array}{l}\text { Pierde una ficha } \\
\text { verde }\end{array}$ & $\begin{array}{l}\text { Pierde una ficha } \\
\text { verde }\end{array}$ \\
\hline & & & $\begin{array}{l}\text { Gana una ficha } \\
\text { verde }\end{array}$ & Nada & $\begin{array}{l}\text { Gana una ficha } \\
\text { verde }\end{array}$ \\
\hline \multirow{5}{*}{ No diferencial } & & & $\mathrm{ND}(+)$ & $\mathrm{ND}(-)$ & $\mathrm{ND}(+/-)$ \\
\hline & & & $\begin{array}{l}\text { Gana una ficha } \\
\text { roja o verde }\end{array}$ & Nada & $\begin{array}{l}\text { Gana una ficha } \\
\text { roja o verde }\end{array}$ \\
\hline & & & Nada & $\begin{array}{l}\text { Pierde una ficha } \\
\text { roja o verde }\end{array}$ & $\begin{array}{l}\text { Pierde una ficha } \\
\text { roja o verde }\end{array}$ \\
\hline & & & Nada & $\begin{array}{l}\text { Pierde una ficha } \\
\text { roja o verde }\end{array}$ & $\begin{array}{l}\text { Pierde una ficha } \\
\text { roja o verde }\end{array}$ \\
\hline & & & $\begin{array}{l}\text { Gana una ficha } \\
\text { roja o verde }\end{array}$ & Nada & $\begin{array}{l}\text { Gana una ficha } \\
\text { roja o verde }\end{array}$ \\
\hline
\end{tabular}


Cada niño participó en una única sesión individual que duró, aproximadamente, unos 30 minutos. El experimento se estructuró en dos fases, una primera fase de pretest, formada por cuatro ensayos de igualación de identidad a la muestra, y ocho ensayos de discriminación condicional o igualación simbólica a la muestra; y una segunda fase de discriminación condicional, formada por 40 ensayos de igualación simbólica, aleatorizados en 10 bloques de 4 ensayos cada uno. La fase de pretest entrenaba la habilidad de los participantes para discriminar los estímulos empleados. En el primer ensayo de igualación a la muestra, el experimentador daba al niño instrucciones verbales. Para prevenir cualquier error por parte del experimentador, las instrucciones fueron leídas por éste en presencia del observador, que previamente fue informado de que debía anotar cualquier desviación significativa respecto del procedimiento a seguir. Las instrucciones se dieron a cada niño diciéndole que iba a jugar a un juego de memoria, de modo que cuando respondiera correctamente ganaría una ficha roja o verde que tenía que depositar en el recipiente de su color correspondiente y/o que si cometía un error se le quitaría una ficha verde o roja, según la condición experimental a la que había sido asignado. Se mostraba entonces al niño la asociación entre las fichas rojas y la comida, y entre las fichas verdes y los juguetes. El participante veía la primera página con el dibujo de un lápiz (o unas tijeras) centrado en la mitad superior de la hoja y dos dibujos en la mitad inferior, un lápiz y unas tijeras, y se le pedía que señalara el dibujo que fuera igual al primero. En el primer ensayo de discriminación condicionada, se le decía que el juego iba a cambiar un poquito, el dibujo que aparecía en la parte superior de la página (estímulo muestra, un rombo o una flor), no sería igual a los dos dibujos presentados en la parte inferior de la página (estímulos comparación, tijera y lápiz), y que primero tenía que adivinar cuál era el dibujo correspondiente y después memorizarlo para la próxima vez. En este caso, el participante tenía que aprender la asociación entre rombo y tijera y entre flor y lápiz (ver Tabla 1).

Las demoras se introdujeron gradualmente de la siguiente manera: los dos primeros ensayos de igualación de identidad y los dos primeros de discriminación condicional no incluían demora. En estos cuatro ensayos, el estímulo muestra y las dos imágenes de comparación aparecían en la misma página. El tercer y cuarto ensayo de igualación de identidad y del tercero al quinto de discriminación condicional, incorporaron una demora de 1 segundo aproximadamente. En estos ensayos, el estímulo muestra estaba en una página y las dos imágenes de comparación en la página siguiente. Los últimos tres ensayos de discriminación condicional de la fase pretest, presentaron demoras de unos 2 segundos. En estos ensayos, aparecía una página en blanco entre la del estímulo muestra y la de los dos de comparación, que servía como demora. Todos los niños superaron el 75\% de aciertos en la fase de pretest como criterio para participar en el experimento.

En la fase de discriminación condicional del Experimento 1 a, se utilizó el mismo procedimiento que en los tres úl- timos ensayos de discriminación condicional de la fase de pretest excepto que en este caso los niños tenían que establecer nuevas asociaciones. En concreto, tuvieron que aprender que la letra griega gamma estaba asociada con la estrella, y que la cruz estaba asociada al círculo. Cada estímulo muestra aparecía dos veces en cada uno de los diez bloques, y el estímulo comparación correcto aparecía el mismo número de veces a la izquierda que a la derecha. Cada ensayo consistió en cuatro páginas, la primera de ellas contenía el número del ensayo escrito en la esquina inferior derecha. La segunda, contenía el estímulo discriminativo, un estímulo muestra centrado en la mitad superior de la página. La tercera era una página en blanco y servía como demora de unos 2 segundos. Por último, la cuarta página contenía dos estímulos de comparación equidistantes respecto de la línea media de la página, situados en la mitad inferior de la misma. La Figura 1 muestra la secuencia de estímulos utilizada. Las respuestas se realizaban señalando el dibujo que ellos pensaban que estaba asociado al estímulo muestra. Una vez emitida la respuesta, se administraba la consecuencia de forma inmediata según la condición experimental a la que habían sido asignados (ver Tabla 1). La fase de discriminación condicional del Experimento 1b era muy similar a la del 1a excepto por los estímulos muestra y comparación utilizados (dos figuras geométricas, una con forma de círculo y otra con forma de espiral, y cuatro combinaciones de flechas) y porque en todos los ensayos aparecían cuatro estímulos de comparación (las combinaciones de flechas), las dos comparaciones correctas y dos distractores muy similares.

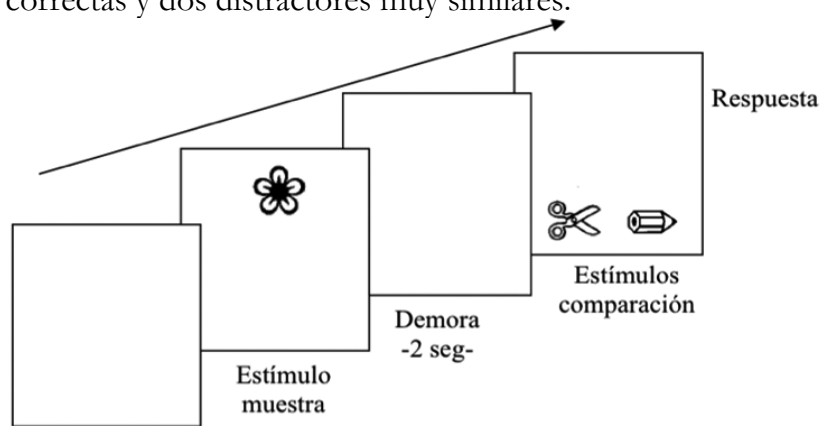

Figura 1. Secuencia de estímulos utilizada en el Experimento 1. La secuencia temporal se representa de izquierda a derecha.

\section{Análisis estadísticos}

Se analizó el porcentaje de aciertos obtenidos en la tarea, agrupándolos en diez bloques de cuatro ensayos cada uno, mediante un Análisis de Varianza (ANOVA) para un diseño factorial de medidas repetidas con dos variables manipuladas entre-sujetos, Consecuencias (diferenciales vs. no diferenciales) y Entrenamiento $(+,-,+/-)$ y una variable manipulada intrasujeto, Bloque de ensayo $(1,2,3,4,5,6,7,8,9$ y 10). Como criterio para el análisis se tomó $p<.05$. Se realizaron las correcciones de Greenhouse-Heisser en los casos en los que se violó el supuesto de esfericidad (prueba de Mauchly). 
Las comparaciones Post hoc fueron realizadas con la prueba LSD de Fisher.

\section{Resultados}

\section{Experimento 1 a}

En el análisis realizado de los datos obtenidos por el grupo de edad de 5 años (ver Figura 2) se observó un efecto estadísticamente significativo del Tipo de entrenamiento $\left[F(2,48)=5.75, p=.006, \eta_{p}^{2}=.19\right]$. El grupo entrenado bajo la condición "-”, registró un porcentaje de respuestas correctas del 69\%, frente al 62\% y 59\% que registraron los grupos “+” y “+/-", respectivamente, encontrándose dife- rencias estadísticamente significativas entre este grupo de entrenamiento $(-)$ y los otros dos $(p s<.05)$. Los resultados muestran también un efecto significativo de Consecuencias, $\left[F(1,48)=66.96, p<.001, \eta_{p}^{2}=.58\right]$, reflejando claramente que bajo la condición diferencial los niños ejecutaron mejor la tarea, con un porcentaje de respuestas correctas del $74 \%$ frente al 53\% obtenido en la condición no diferencial. Se observa, además, un efecto significativo del Bloque de ensayos $\left[F(6.4,305.6)=5.02, p<.001, \eta_{p}{ }^{2}=.09\right]$ que reflejó una mejor ejecución al ir avanzando los bloques de ensayos (48\%, $59 \%, 59 \%, 58 \%, 66 \%, 68 \%, 74 \%, 68 \%, 66 \%$ y $69 \%$ de respuestas correctas en los bloques de ensayos 1, 2, 3, 4, 5, 6, 7, 8,9 y 10 , respectivamente).
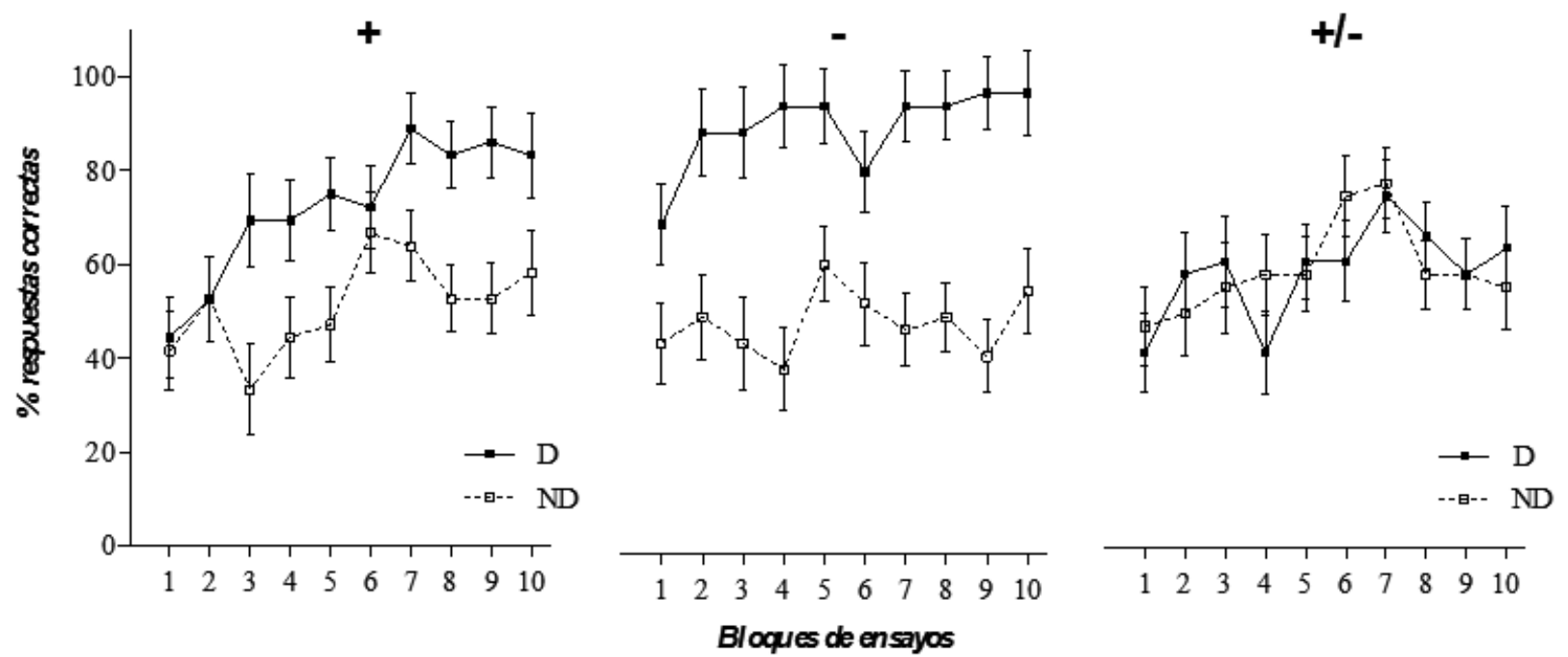

Figura 2. Porcentaje medio de respuestas correctas obtenidos por los participantes del Experimento 1a en función del Tipo de entrenamiento (“+”, “_“ y "+/-"), Consecuencias (diferenciales: D, no diferenciales: ND) y Bloque de ensayos (10 bloques de 4 ensayos cada uno).

Por último, la interacción Tipo de entrenamiento $\times$ Consecuencias fue significativa $\left[F(2,48)=23.03, p<.001, \eta_{p}{ }^{2}=\right.$ .49]. El análisis de esta doble interacción reveló que en los grupos de entrenamiento "-” y "+” había diferencias significativas entre las condiciones diferencial y no diferencial, encontrándose una mejor ejecución en ambos grupos al administrar consecuencias diferenciales $(73 \%$ vs. $51 \%$ y $90 \%$ vs. $49 \%$ en las condiciones diferencial y no diferencial para los grupos “+” y “-”, respectivamente) $[F(1,16)=18.86, p=$ $.001, \eta_{p}^{2}=.54$ en el grupo “+” y $F(1,16)=142.77, p<$ $.001, \eta_{p}^{2}=.90$ en el grupo “-”]. En el grupo “+/-", no se observaron diferencias significativas entre ambas condiciones $(p>$.05). No hubo ningún otro efecto ni interacción significativas $(p s>.05)$. El análisis del primer bloque de ensayos tampoco reveló diferencias significativas entre las condiciones diferencial y no diferencial lo que indica que todos los niños partieron de un mismo nivel de ejecución $(p>.05)$.

\section{Experimento $1 b$}

Los resultados del análisis de los datos obtenidos por los niños de 7 años de edad (ver Figura 3) mostraron un efecto significativo de Consecuencias $[F(1,30)=40.64, p<.001$, $\left.\eta_{p}^{2}=.58\right]$ reflejando, de nuevo, que los niños realizaron mejor la tarea bajo la condición diferencial $(80 \%$ vs. $47 \%$ para la condición diferencial y no diferencial, respectivamente). Se observó también un efecto significativo del Bloque de ensayos $\left[F(5.4,162.9)=15.22, p<.001, \eta_{p}^{2}=.34\right]$ debido a que los niños mostraron una mejor ejecución al ir avanzando los bloques de ensayos $(42 \%, 45 \%, 55 \%, 56 \%, 66 \%, 63 \%, 72 \%$, $73 \%, 78 \%$ y $83 \%$ de respuestas correctas en los bloques de ensayos $1,2,3,4,5,6,7,8,9$ y 10 , respectivamente). 

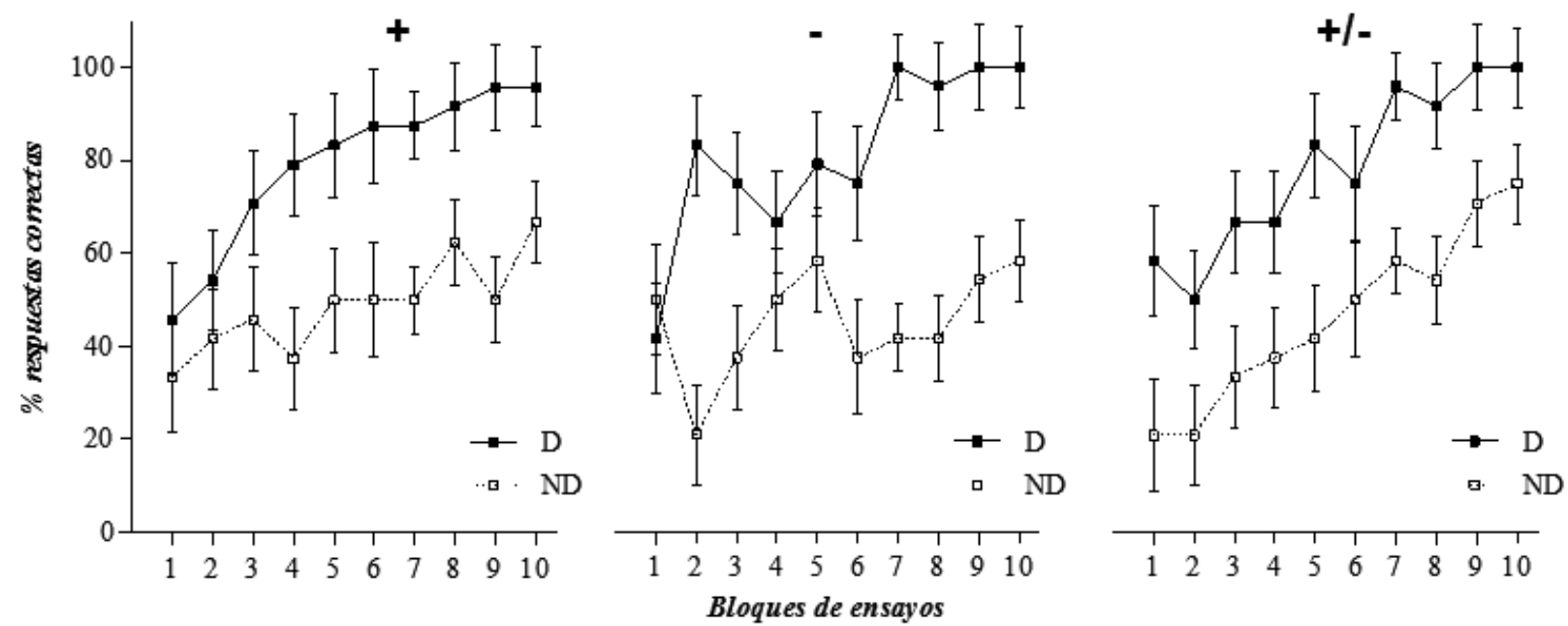

Figura 3. Porcentaje medio de respuestas correctas obtenidos por los participantes del Experimento 1b en función del Tipo de entrenamiento (“+”, “_“ y "+/-"), Consecuencias (diferenciales: D, no diferenciales: ND) y Bloque de ensayos (10 bloques de 4 ensayos cada uno).

No se encontró ningún otro efecto ni interacción significativas $(p s>.05)$. Al igual que en el Experimento 1a, el análisis del primer bloque de ensayos no mostró ninguna diferencia significativa entre ambas condiciones, diferencial y no diferencial $(p>.05)$.

\section{Discusión}

El objetivo principal del presente experimento fue explorar si distintas formas de administrar las consecuencias influirían en el efecto que el procedimiento de consecuencias diferenciales tiene en el aprendizaje discriminativo utilizando una tarea de lápiz y papel. Tal y como encontraron previamente Martínez et al. (2009, 2013) los niños de menor edad aprendieron mejor la tarea cuando fueron asignados al grupo “-”, es decir, cuando perdían reforzadores tras sus respuestas incorrectas. Sin embargo, esta diferencia desapareció en los niños de 7 años de edad (el grupo entrenado bajo la condición "-" registró un porcentaje de respuestas correctas del $63 \%$, frente al 64\% y 63\% que registraron los grupos “+” y “+/-", respectivamente).

De especial relevancia es que en ambos grupos de edad se encontró, en general, que los niños realizaban mejor la tarea cuando las consecuencias eran administradas de forma diferencial en comparación a cuando dichas consecuencias eran aleatorias. Este efecto fue modulado en el grupo de 5 años (Experimento 1a) por el tipo de entrenamiento utilizado de tal forma que se observó cuando los niños recibían reforzadores tras las respuestas correctas (grupo “+”) o se retiraban tras las incorrectas (grupo "-”), pero desaparecía (es decir, la ejecución era similar bajo condiciones diferenciales y no diferenciales) en el grupo mixto (aquellos que recibían reforzadores tras las respuestas correctas y los perdían tras las incorrectas). En el grupo de 7 años el efecto beneficioso del PCD sobre el aprendizaje discriminativo se observó en todos los casos (grupos “+”, “-”y “+/-”). Estos resultados son similares a los encontrados por Martínez et al. (2009, 2013). Tal y como estos autores sugieren, creemos que el tipo de reforzamiento "+/-" fue muy confuso para el grupo de niños de 5 años quienes no terminaban de entender por qué en algunas ocasiones perdían fichas de colores y en otras las ganaban. De hecho, el porcentaje de aciertos obtenidos en ambas condiciones fue muy bajo (59\% vs. 59\% bajo consecuencias diferenciales y no diferenciales, respectivamente). Al igual que Martínez et al. (2009, 2013), creemos que el efecto volvería a aparecer con un procedimiento más sencillo, por ejemplo, al utilizar directamente reforzadores tangibles que los niños pudiesen ganar o perder. Esta hipótesis la pondremos a prueba en el siguiente experimento el cual nos servirá para comprobar si los resultados obtenidos hasta el momento se verían modulados por el tipo de consecuencias administradas. En concreto, en el Experimento 2 utilizaremos sólo reforzadores primarios (chocolatinas y estampas) manteniendo sin cambios el resto de condiciones experimentales.

\section{Experimentos 2a y $2 b$}

\section{Método \\ Participantes}

Al igual que en el Experimento 1, todos los niños que participaron en el estudio eran alumnos del C.E.I.P. Lope de Vega (Almería, España). En el Experimento 2a participaron 40 niños con edades comprendidas entre 4 años y 11 meses y 5 años y 11 meses (19 niños y 21 niñas) y en el Experimento $2 \mathrm{~b}, 40$ niños de entre 6 años y 11 meses y 7 años y 11 meses años de edad (21 niños y 19 niñas). Todos los niños tenían visión normal o corregida a la normalidad. 


\section{Estimulos y materiales}

La forma de administrar la tarea, el lugar, los estímulos y materiales utilizados fueron idénticos a los empleados en el Experimento 1 (a y b) excepto que como consecuencias se utilizaron sólo reforzadores primarios (chocolatinas lacasitos- y estampas). El modo de registrar los datos fue el mismo que en el experimento anterior contándose, también, con la presencia de un segundo observador.

\section{Procedimiento}

El procedimiento seguido en el Experimento 2 fue similar al del experimento anterior. La diferencia, como señalábamos en el apartado anterior, consistió en que tras una respuesta correcta los niños recibían (o se les quitaba) un reforzador primario (comida o juguetes). Los participantes también se dividieron en dos grupos según su edad, 5 años (Experimento 2a) y 7 años (Experimento $2 \mathrm{~b}$ ) recibiendo consecuencias diferenciales la mitad de los niños de cada grupo y consecuencias no diferenciales la otra mitad.

\section{Análisis estadísticos}

Al igual que en el Experimento 1, se analizó el porcentaje de aciertos obtenidos en la tarea, agrupándolos en diez bloques de cuatro ensayos cada uno, mediante un Análisis de Varianza (ANOVA) para un diseño factorial de medidas re- petidas con dos variables manipuladas entre-sujetos, Consecuencias (diferenciales $v s$. no diferenciales) y Entrenamiento $(+,-,+/-)$ y una variable manipulada intrasujeto, Bloque de ensayos $(1,2,3,4,5,6,7,8,9$ y 10).

\section{Resultados}

\section{Experimento $2 a$}

El análisis de los datos obtenidos por el grupo de edad de 5 años (ver Figura 4) reveló un efecto estadísticamente significativo del Tipo de entrenamiento $[F(2,34)=3.72, p=$ $\left..035, \eta_{p}^{2}=.18\right]$ debido a que el grupo entrenado bajo la condición "_" (78\% de respuestas correctas) mostró una mejor ejecución en la tarea que aquellos entrenados en los grupos “+” y "+/-" (68\% y 68\% de exactitud, respectivamente; $p s<$ $.05)$. Al igual que en el experimento anterior, también se observó un efecto significativo de Consecuencias $[F(1,34)=$ $\left.34.55, p<.001, \eta_{p}^{2}=.50\right]$ y de Bloque de ensayos $[F(9,306)$ $\left.=5.86, p<.001, \eta_{p}^{2}=.15\right]$ indicando que, en general, aquellos en la condición diferencial realizaron mejor la tarea $(82 \%$ $v$ s. $61 \%$ de respuestas correctas para las condiciones diferencial y no diferencial, respectivamente) y que todos mostraron una mejor ejecución en la misma a medida que avanzaban los ensayos $(56 \%, 64 \%, 62 \%, 73 \%, 72 \%, 80 \%, 80 \%, 71 \%$, $78 \%$ y $77 \%$ de exactitud en los bloques de ensayos 1, 2, 3, 4, $5,6,7,8,9$ y 10 respectivamente). No hubo ningún otro efecto ni interacción significativas ( $p s>.05)$.
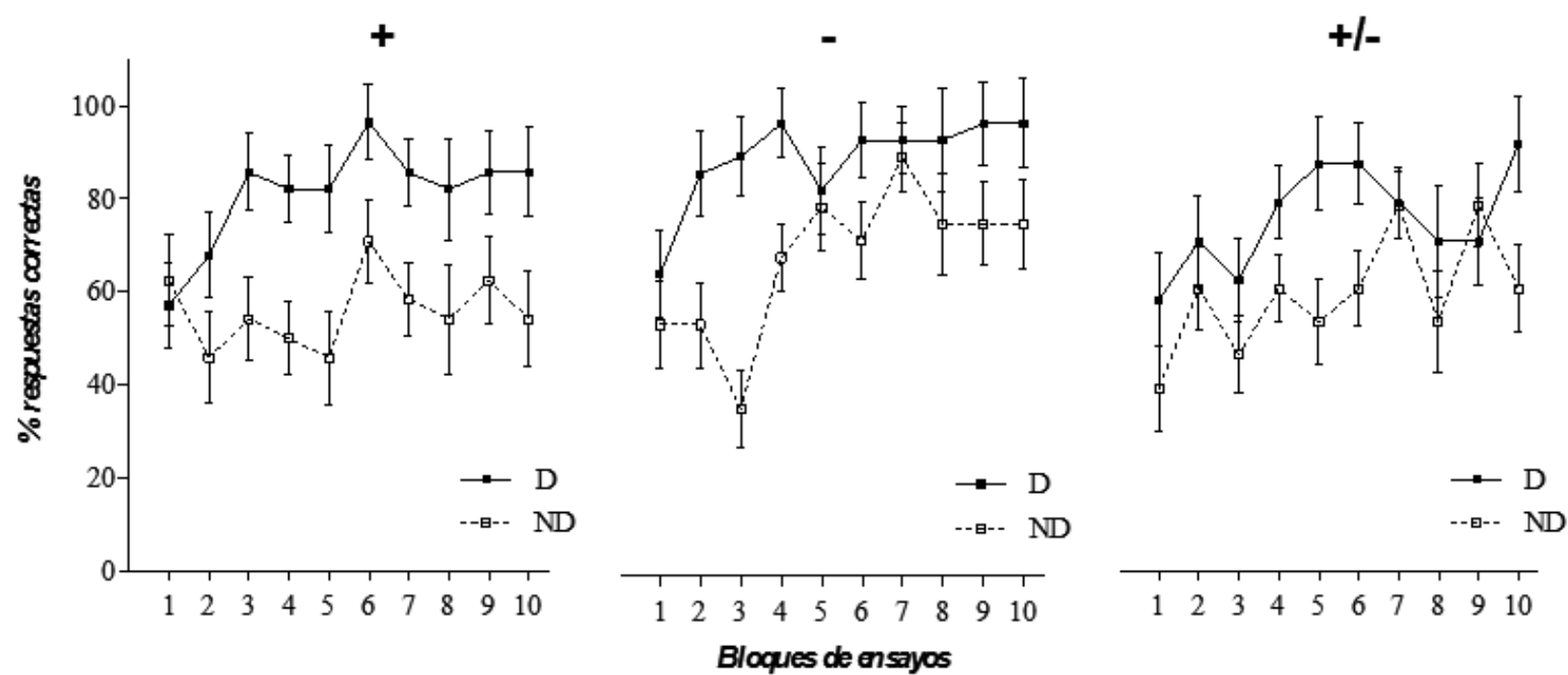

Figura 4. Porcentaje medio de respuestas correctas obtenidos por los participantes del Experimento 2a en función del Tipo de entrenamiento (“+”, “-“y "+/-"c), Consecuencias (diferenciales: D, no diferenciales: ND) y Bloque de ensayos (10 bloques de 4 ensayos cada uno).

\section{Experimento $2 b$}

Los resultados del análisis de los datos obtenidos por los niños de 7 años de edad (ver Figura 5) mostraron que el grupo entrenado bajo consecuencias diferenciales obtuvo un mayor porcentaje de respuestas correctas que aquellos en- trenados con consecuencias no específicas (83\% vs. 54\% de exactitud para las condiciones diferencial y no diferencial, respectivamente) $\left[F(1,34)=39.43, p<.001, \eta_{p}^{2}=.54\right]$. También se encontró un efecto significativo de Ensayos $\left[F(6.1,206.4)=21.92, p<.001, \eta_{p}^{2}=.39\right]$ el cual se vio modulado por la doble interacción significativa Ensayo $\times$ Con- 
secuencias $\left[F(6.1,206.4)=2.56, p=.020, \eta_{p}^{2}=.07\right]$. El análisis de la interacción reveló que aunque los niños en ambas condiciones mostraban un aprendizaje de la tarea, la forma del mismo varió en función de la condición. En concreto, a partir del bloque 5 la ejecución no difiere con respecto a la obtenida en el último bloque de ensayos en la condición diferencial $(41 \%, 66 \%, 84 \%, 82 \%, 91 \%, 86 \%, 91 \%, 93 \%, 96 \%$ y $95 \%$ de exactitud para los ensayos $1,2,3,4,5,6,7,8,9$ y 10 , respectivamente), mientras que en la condición no diferencial hay una mayor variabilidad desapareciendo dicha diferencia en el bloque 7 y apareciendo nuevamente en el 8 siendo esta marginalmente significativa $(p=.07 ; 29 \%, 39 \%$, $36 \%, 52 \%, 47 \%, 59 \%, 65 \%, 61 \%, 75 \%$ y $77 \%$ de exactitud para los ensayos $1,2,3,4,5,6,7,8,9$ y 10 , respectivamente). Es decir, a diferencia de aquellos en la condición no diferencial que parecen continuar aprendiendo la tarea hasta los últimos ensayos, el grupo diferencial alcanza la asíntota del aprendizaje en el bloque 5 a partir del cual su ejecución tiende a estabilizarse. Esto se ve confirmado por el componente cuadrático significativo en el análisis de tendencia para la condición diferencial $\left[F(1,19)=39.18, p<.001, \eta_{p}{ }^{2}=.67\right]$ pero no para la no diferencial $(F<1)$. Cabe destacar que no hubo diferencias significativas entre ambas condiciones al analizar por separado el primer bloque de ensayos $(p>.05)$. No se encontró ningún otro efecto ni interacción significativas $(p s>.05)$.
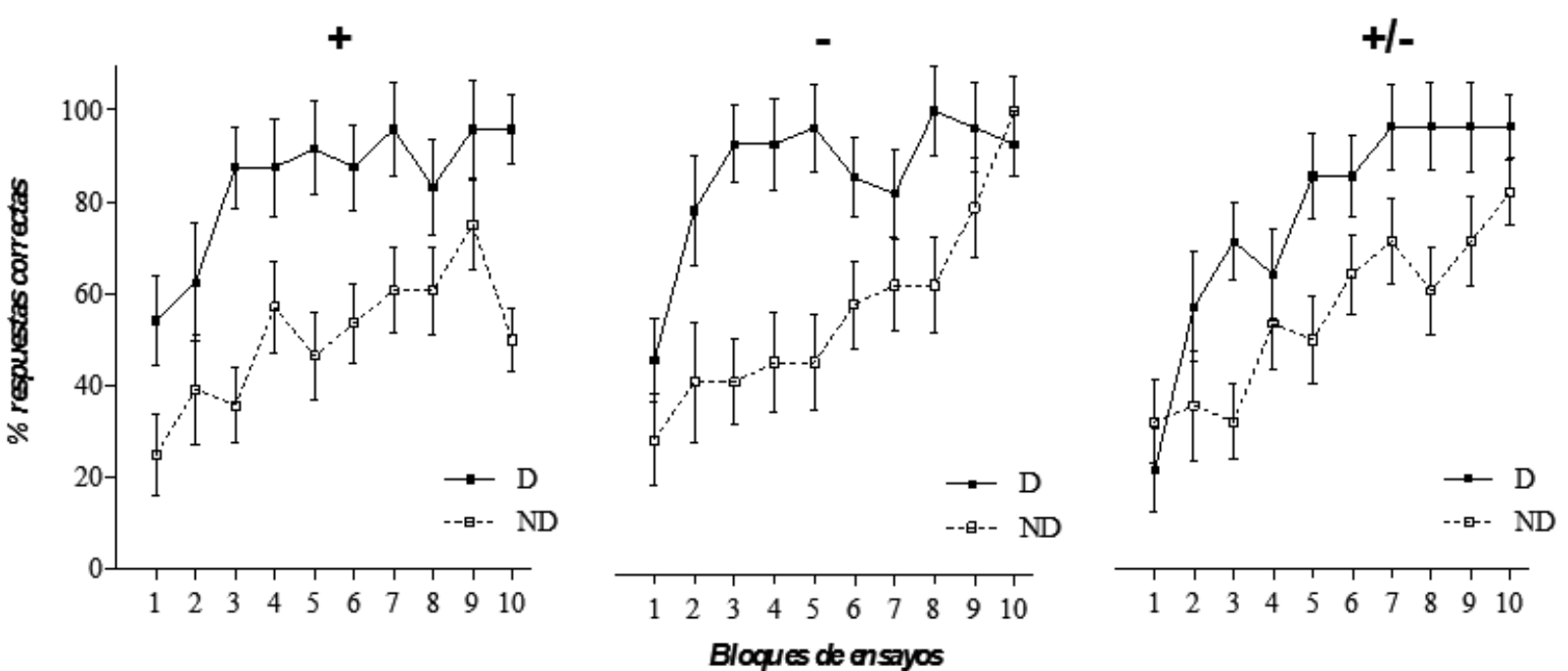

Figura 5. Porcentaje medio de respuestas correctas obtenidos por los participantes del Experimento 2b en función del Tipo de entrenamiento (“+”, “-_ y "+/-"), Consecuencias (diferenciales: D, no diferenciales: ND) y Bloque de ensayos (10 bloques de 4 ensayos cada uno).

\section{Discusión}

Los resultados encontrados en ambos experimentos son muy similares a los obtenidos en los dos experimentos anteriores (1a y 1b). Es decir, nuevamente se observó (i) que los niños de menor edad aprendieron mejor la tarea cuando fueron asignados al tipo de entrenamiento "_,", algo que no ocurrió en el grupo de 7 años en el que los tres tipos de entrenamiento fueron igualmente eficaces, y (ii) que todos los niños realizaron mejor la tarea cuando las consecuencias fueron administradas de forma diferencial en comparación a cuando dichas consecuencias eran aleatorias. Sin embargo, a diferencia de lo observado en el Experimento 1a en el que la forma de administrar las consecuencias (diferencial $v$ s. no diferencial) no influyó en la ejecución del grupo de cinco años de edad cuando fueron entrenados en la condición "+ /-", y en consonancia con la hipótesis que planteábamos previamente, el efecto beneficioso del PCD sobre el aprendizaje discriminativo se observó en todos los casos (grupos “+”, “" y “+/-") en ambos grupos de edad.

\section{Discusión General}

El objetivo general del presente estudio fue explorar, en una muestra de niños con edades comprendidas entre 5 y 7 años, si el efecto beneficioso que tiene la utilización de consecuencias diferenciales en el aprendizaje discriminativo es independiente no sólo del tipo de consecuencias y estímulos utilizados sino, también, del modo en el que se administran y presentan éstos. Para examinar esta hipótesis, a partir de una tarea de igualación simbólica demorada a la muestra similar a la utilizada en estudios previos por Martínez et al. (2009, 2013), se diseñaron dos experimentos iguales entre sí, excepto en el hecho de que en el segundo de ellos se emplearon como consecuencias únicamente reforzadores primarios. Lo relevante, y lo que diferencia al presente estudio de los realizados previamente por Martínez y colaboradores (2009, 2013), es que dicha tarea se presentó en formato papel y no informatizada tal y como ha sido habitual en los estudios publicados en los últimos años (p.e., Estévez et al., 2007; Martínez et al., 2012; Mok y Overmier, 2007; Mok et al., 2009). 
Los resultados obtenidos en el Experimento 1 mostraron que tanto los niños de 5 años de edad (Experimento 1a) como los de 7 (Experimento 1b) ejecutaron mejor la tarea cuando las consecuencias eran administradas de forma diferencial frente a la administración de consecuencias aleatorias. Además, tal y como observaron previamente Martínez et al. (2009, 2013), dicha mejora en la ejecución fue, en general, independiente del tipo de entrenamiento utilizado. Así, el efecto beneficioso del PCD sobre el aprendizaje discriminativo se hizo palpable en el grupo "+” y en el grupo "-." en los niños de menor edad, observándose en todos los grupos (“+”, “-” y “+/-”) en los niños mayores. Estos resultados parecen explicarse atendiendo a que el tipo de reforzamiento "+/-" (en el que en algunas ocasiones perdían fichas de colores y en otras ganaban) resultó difícil de comprender para el grupo de 5 años. En el Experimento 2, en el que se utilizó un procedimiento más sencillo caracterizado por el uso de reforzadores primarios que los niños podían ganar o perder en el mismo instante, los resultados fueron similares a los observados en el Experimento 1a y en el Experimento 1b. Esto es, tal y como esperábamos en base a los resultados obtenidos previamente por Martínez et al. (2009, 2013), se encontró que la utilización del PCD mejoró la ejecución en ambos grupos de edad (Experimento 2a -5años- y Experimento $2 \mathrm{~b}-7$ años-) bajo todas las condiciones de entrenamiento (“+", "-" y "+/--").

Cabe destacar que la mejora sobre el aprendizaje discriminativo observada en ambos grupos de edad en la condición diferencial no se ve influenciada por el hecho de que las consecuencias específicas inmediatas tras las respuestas sean o no reforzadores primarios. Lo significativo de este hallazgo es que el PCD puede aplicarse como una herramienta útil para mejorar el aprendizaje discriminativo en diferentes contextos (p.e., colegios, centros de atención temprana, residencias, etc.) en los que se podría implementar un sistema de fichas administradas de forma diferencial que, transcurrido un tiempo, podrían ser canjeadas por diferentes reforzadores.

\section{Referencias}

Easton, A. (2004). Differential reward outcome learning in adult humans. Behavioural Brain Research, 154, 165-169. doi: 10.1016/j.bbr.2004.02.023

Estévez, A. F. (2005). The differential outcomes effect: a useful tool to improve discriminative learning in humans. The Behavior Analyst Today, 6, 216-220.

Estévez, A. F. y Fuentes, L. J. (2003). Differential outcomes effect in fouryear- old children. Psicológica, 24, 159-167. Recuperado de http://www.uv.es/revispsi

Estévez, A. F., Fuentes, L. J., Mari-Beffa, P., González, C. y Álvarez, D. (2001). The differential outcomes effect as useful tool to improve conditional discrimination learning in children. Learning and Motivation, 1(32), 48-64. doi: 10.1006/lmot.2000.1060

Estévez, A. F., Overmier, J. B., Fuentes, L. J. y González, C. (2003). Differential outcomes effect in children and adults with Down syndrome. American Journal of Mental Retardation, 108(2), 108-116. doi: 10.1352/0895-8017(2003)108

Estévez, A. F., Vivas, A. B., Alonso, D., Marí-Beffa, P., Fuentes, L. J. y Overmier, J. B. (2007). Enhancing challenged students' recognition of mathematical relations through differential outcomes training. The Quar-
Asimismo, estos datos permiten pensar en la posibilidad de utilizar como reforzadores para futuros estudios tickets para una rifa o sorteo final o únicamente reforzadores secundarios (p.e., López-Crespo, Plaza, Fuentes y Estévez, 2009; Mok y Overmier, 2007; Plaza, Estévez, López-Crespo y Fuentes, 2011).

Finalmente, otro aspecto muy relevante del presente estudio es que se observa un beneficio del PCD independientemente del medio o soporte utilizado tanto para la presentación de los estímulos muestra y comparación (en este caso en formato papel), como para la administración de consecuencias, primarias y/o secundarias (que fueron administradas directamente por el experimentador). Así, el efecto beneficioso que tiene la utilización de consecuencias diferenciales sobre el aprendizaje discriminativo no sólo es independiente del tipo de consecuencias y del tipo de entrenamiento empleado, sino también, del medio utilizado para presentar los estímulos y las consecuencias. Esto nos lleva a sugerir nuevamente la potencial utilidad de este procedimiento en diferentes contextos y campos de actuación, dada la facilidad y accesibilidad que supone el formato papel. Pensemos, por poner un ejemplo, que el PCD podría ayudar a niños en edad escolar a discriminar diferentes símbolos ma-

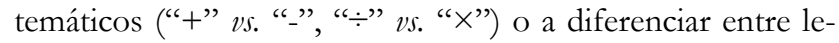
tras similares ("p" vs. "d", "b" vs. "d"), lo que sería muy útil especialmente en niños con dislexia.

Agradecimientos: Esta investigación se ha realizado con fondos de los proyectos PSI2012-39228 financiado por el Ministerio de Economía y Competitividad (España) y DPI 06-2014 financiado por la Universidad Autónoma de Chile (Chile).

Deseamos agradecer a todos los miembros del C.E.I.P. Lope de Vega que han ayudado a realizar el presente estudio, así como a los padres o tutores quienes, tras ser debidamente informados, han dado su autorización para que los niños puedan participar en el mismo.

terly Journal of Experimental Psychology, 60(4), 571-580. doi: 10.1080/17470210600820039

Joseph, B., Overmier, J. B. y Thompson, T. (1997). Food and nonfood related differential outcomes in equivalence learning by adults with Prader-Willi syndrome. American Journal of Mental Retardation, 4, 374-386. Recuperado de http://www.researchgate.net

Legge, E. L. G. y Spetch, M. L. (2009). The differential outcomes effect (DOE) in spatial localization: An investigation with adults. Learning and Motivation, 40, 313-328. doi: 10.1016/j.lmot.2009.03.002

Lewis, M. D., Lamm, C., Segalowith, S. J., Stieben, J. y Zelazo, P. D. (2006). Neurophysiological correlates of emotion regulation in children and adolescents. Journal of Cognitive Neuroscience, 18(3), 430-443. doi: 10.1162/jocn.2006.18.3.430

López-Crespo, G. y Estévez, A. F. (2013). Working memory improvement by the differential outcomes procedure. En S.H. Clair-Thompson (Ed.), Working memory: Developmental differences, component processes, and improvement mechanism (pp. 145-157). New-York, NY: Nova Publishers.

López-Crespo, G., Plaza, V., Fuentes, L. J. y Estévez, A. F. (2009). Improvement of age-related memory deficits by differential outcomes. International Psychogeriatrics, 21, 503-510. doi: 10.1017/S1041610209008576 
Maki, P., Overmier, J. B., Delos, S. y Gutman, A. J. (1995). Expectancies as factors influencing conditional discrimination performance of children. The Psychological Record, 45, 45-71. Recuperado de http:/ http://psycnet.apa.org/psycinfo

Martínez, L., Estévez, A. F., Fuentes, L. J. y Overmier, J. B. (2009). Improving conditional discrimination learning and memory in fie-year-old children: DOE using different types of reinforcement. The Quarterly Journal of Experimental Psychology, 62(8), 1617-1630. doi: 10.1080/17470210802557827

Martínez, L., Flores, P., González-Salinas, C., Fuentes, L. J. y Estévez, A. F. (2013). The effects of differential outcomes and different types of consequential stimuli on seven-year-old children's discriminative learning and memory. Learning and Behavior, 41, 298-308. doi: 10.3758/s13420013-0105-y

Martínez, L., Marí-Beffa, P., Roldán-Tapia, D., Ramos-Lizana, J., Fuentes, L. J. y Estévez, A. F. (2012). Training with differential outcomes enhances discriminative learning and visuospatial recognition memory in children born prematurely. Researh in Developmental Disabilities, 33, 76-84. doi: 10.1016/j.ridd.2011.08.022

Miller, O. T., Waugh, K. M. y Chambers, K. (2002). Differential outcomes effect: increased accuracy in adults learning kanji with stimulus specific rewards. The Psychological Record, 52, 315-324. Recuperado de http://opensiuc.lib.siu.edu/

Mok, L. W., Estévez, A. F. y Overmier, J. B. (2010). Unique outcome expectations as a training and pedagogical tool. Psychological Record, 60(2), 227248. Recuperado de http:/ http://opensiuc.lib.siu.edu/
Mok, L. W. y Overmier, J. B. (2007). The differential outcomes effect in normal human adults using a concurrent-task within-subjects design and sensory outcomes. The Psychological Record, 57, 187-200. Recuperado de http://opensiuc.lib.siu.edu/

Mok, L. W., Thomas, K. M., Lungu, O. V. y Overmier, J. B. (2009). Neural correlates of cue-unique outcome expectations under differential outcomes training: An fMRI study. Brain Research, 1265, 111-127. doi: 10.1016/j.brainres.2008.12.072

Peterson, G. B. y Trapold, M. A. (1980). Effects of altering outcome expectancies on pigeons' delaved conditional discrimination performance. Learning and Motivation, 11, 267-288. doi: 10.1016/0023-9690(80)90001-6

Plaza, V., Estévez, A. F., López-Crespo, G. y Fuentes, L. J. (2011). Enhancing recognition memory in adults through differential outcomes. Acto Psychologica, 136, 129-136. doi: 10.1016/j.actpsy.2010.11.001

Posner, M. I. y Rothbart, M. K. (2009). Toward a physical basis of attention and self-regulation. Physics of Life Reviews, 6, 103-120. doi: 10.1016/j.plrev.2009.02.001

Trapold, M. A. (1970). Are expectancies based upon different positive reinforcing events discriminably different? Learning and Motivation, 1, 129140. doi: 10.1016/0023-9690(70)90079-2

Urcuoli, P. J. (2005). Behavioral and associative effects of differential outcomes in discrimination learning. Learning \& Behavior, 33, 1-21. doi: 10.3758/BF03196047

Walters, R. H. y Parke, R. D. (1964). Arousal, isolation, and discrimination learning in children. Journal of Experimental Child Psychology, 1, 163-173. doi: 10.1016/0022-0965(64)90018-9

(Articulo recibido: 09-02-2015; revisado: 21-05-2015; aceptado: 26-05-2015) 